Die Abendveranstaltung fand im Meistersaal Berlin statt und wurde von zwei Cellisten musikalisch umrahmt. Sie verstanden es auf sehr humorvolle Art und Weise, die Themen Controlling und Reporting in die Darbietung zu integrieren.
So bekamen die Zuhörer die "Zauberflöte“ in einer einzigen Minute sozusagen als „Executive Summary“ präsentiert.

Nicolás Wiedemann, Vallendar

\title{
Anwendungsorientiertes Controlling in Forschung und Lehre
}

\section{Jahrestagung des Arbeitskreises Controlling (AKC) an der Hochschule Bonn-Rhein-Sieg, Sankt Augustin}

Die dreitägige Jahrestagung des „Arbeitskreises Controlling an Hochschulen für angewandte Wissenschaften und Fachhochschulen“ (AKC) fand vom 22.05. bis 24.05.2014 an der Hochschule Bonn-Rhein-Sieg statt. 32 Teilnehmer aus Deutschland und Österreich tauschten sich in Sankt Augustin zu aktuellen Fragen der Controlling-Forschung, -Praxis und -Lehre aus.

Mit Professor Thomas Reichmann startete der erste „Forschungstag“ der Konferenz mit einem hochkarätigen Referenten, der die deutschsprachige Controlling-Lehre und -Forschung in den letzten Jahrzehnten maßgeblich mitgeprägt hat Dass Forschung auch ein wichtiges Thema für den AKC ist, zeigte die intensive Diskussion eines Positionspapiers zur anwendungsorientierten Forschung an Hochschulen für angewandte Wissenschaften und Fachhochschulen. Die Teilnehmer wiesen auf die Relevanz der Forschung als Fundament für eine qualitativ hochwertige Lehre und für den Technologie- und Wissenstransfer in die Praxis hin. Allerdings konstatierten sie auch einen Mangel an praxisrelevanter Controlling-Forschung, der Chancen für eine Positionierung forschungsaffiner Professoren an Hochschulen für angewandte Wissenschaften und Fachhochschulen bietet, ohne dabei die notwendige wissenschaftliche Qualität einzuschränken. Außerdem wurden Ansatzpunkte für gemeinsame Forschungsaktivitäten der Tagungsteilnehmer identifiziert und Möglichkeiten zur Publikation von Forschungsergebnissen erarbeitet. Ausgehend von dem Positionspapier sind weitere Impulse für die anwendungsorientierte Controlling-Forschung in Deutschland zu erwarten.

An den folgenden Tagen stand außerdem die Auseinandersetzung mit der Controlling-Praxis am Beispiel von Postbank AG und Bundeswehr auf dem Programm. Faszinierend war aus Sicht der Teilnehmer das einzigartige und komplexe Geschäftsmodell der Postbank AG als Bestandteil des Deut-
sche-Bank-Konzerns und dessen Implikationen für die praktische Umsetzung des Controllings. Anhand des strategischen Controllings bei der Bundeswehr wurden die besonderen Anforderungen an die Konzeption und Umsetzung von Controlling-Systemen in öffentlichen Organisationen diskutiert.

Außerdem wurden Workshops zu verschiedenen Facetten der Controlling-Lehre wie z. B. zu unterschiedlichen Controlling-Kompetenzen in Bachelor- und Master-Studiengängen sowie zum Einsatz und zur Weiterentwicklung von Planspielen in der Controlling-Lehre durchgeführt. Auch Tipps und Tricks der Controlling-Lehre wurden ausgetauscht. Neben der Arbeit mit Fallstudien wurden insbesondere innovative Lehrund Lernkonzepte wie z. B. die Durchführung von PeerReview-Verfahren mit Studierenden im Rahmen von Seminaren oder Möglichkeiten der Online-Unterstützung diverser Veranstaltungsformate diskutiert. Da Anwendungen moderner Medien wie Abstimmsysteme und Spiele-Apps unter anderem zur Steigerung der Motivation der Studierenden eingesetzt werden können, erhielten die Teilnehmer einen Einblick in Ansätze der „Gamification“ in der ControllingLehre. Zudem erfolgte eine Auseinandersetzung mit der Bedeutung kultureller Kompetenzen für das Controlling. Die Tagung klang mit einer Führung der Teilnehmerinnen und Teilnehmer durch die repräsentativen und privaten Räume des alten Kanzlerbungalows in Bonn aus.

Mit der Nordakademie steht bereits die veranstaltende Hochschule für das Folgejahr fest. Die nächste AKC-Jahrestagung wird vom 28.05. bis 30.05.2015 in der Räumlichkeiten der Hochschule in den Hamburger Docklands stattfinden.

Prof. Dr. Ute Vanini, Fachhochschule Kiel und Sprecherin des AKCs,

Prof. Dr. Andreas Wiesehahn, Hochschule Bonn-Rhein-Sieg 